\title{
A HYBRID METHOD FOR INVERSE BOUNDARY VALUE PROBLEMS FOR AN INCLUSION IN SEMI-INFINITE TWO-DIMENSIONAL DOMAINS
}

\author{
ROMAN CHAPKO AND NADIYA VINTONYAK
}

\begin{abstract}
We consider an inverse Dirichlet boundary value problem for the Laplace equation that consists of the reconstruction of the shape of a bounded domain contained within a semi-infinite region from Cauchy data observed on a part of the infinite boundary. The numerical solution of this problem is obtained by a hybrid method with the corresponding integral equations of the first kind derived by a Green's function technique. To solve the integral equations we use Tikhonov regularization with sinc and trigonometric quadratures for integrals with various singularities. The numerical examples illustrate the feasibility of the hybrid method in the case of $2 D$ semi-infinite regions.
\end{abstract}

1. Introduction. In nondestructive testing, one tries to assess the interior structure of an object from some information measured on the accessible boundary. Such problems are of particular interest for the case of unbounded domains. The mathematical modeling of thermal or electrostatic imaging methods in nondestructive testing and evaluation leads to inverse boundary value problems for the Laplace equation. In principle, in these applications inclusions or interior cracks are detected from overdetermined Cauchy data on the accessible part of the boundary $[\mathbf{1}, \mathbf{2}, \mathbf{1 4}]$.

For simplicity of our presentation we assume that $D_{1} \subset \mathbf{R}^{2}$ is a semiinfinite region with boundary $\Gamma$ and $D_{0}$ is a simply connected bounded domain in $\mathbf{R}^{2}$ with boundary $\Gamma_{0} \in C^{2}$ such that $\bar{D}_{0} \subset D_{1}$. Further, we denote $D:=D_{1} \backslash \bar{D}_{0}$.

Keywords and phrases. Laplace equation, semi-infinite region, inverse boundary value problem, Green's function, integral equation of the first kind, collocation method, trigonometric interpolation, sinc approximation, hybrid method, Tikhonov regularization.

Received by the editors on December 19, 2005, and in revised form on June 27, 2006.

Copyright (C)2007 Rocky Mountain Mathematics Consortium 
For a given bounded continuous function $f$ on $\Gamma$, we consider the Dirichlet problem for the bounded function $u \in C^{2}(D) \cap C(\bar{D})$ satisfying the Laplace equation

$$
\Delta u=0 \text { in } D
$$

and the boundary conditions

$$
\begin{aligned}
& u=0 \text { on } \Gamma_{0} \text { and } \\
& u=f \text { on } \Gamma .
\end{aligned}
$$

Existence and uniqueness of classical or weak solutions for the direct boundary value problem (1.1)-(1.3) are well established, for example, in [8]. We assume that the function $f$ is smooth enough to ensure the existence of the normal derivative of the solution $u$ on $\Gamma$.

Now we shall consider the following inverse problem: determine the boundary $\Gamma_{0}$ from the knowledge of the normal derivative

$$
\frac{\partial u}{\partial \nu}=\phi \quad \text { on } \quad \Sigma
$$

under the assumption that $f \neq 0$. Here $\nu$ is the outward unit normal on $\Gamma, \Sigma \subset \Gamma$ is a subset with a nonempty interior and $\phi$ is a given (measured) function.

The issue of unique reconstruction of the unknown boundary curve $\Gamma_{0}$ from the Cauchy data on $\Sigma$ is settled through the following theorem.

Theorem 1.1. Let $\Gamma_{0}$ and $\widetilde{\Gamma}_{0}$ be two closed curves contained in the semi-infinite region $D_{1}$, and denote by $u$ and $\widetilde{u}$ the solutions to the Dirichlet problem (1.1)-(1.3) for the interior boundaries $\Gamma_{0}$ and $\widetilde{\Gamma}_{0}$, respectively. Assume that $f \neq 0$ and

$$
\frac{\partial u}{\partial \nu}=\frac{\partial \widetilde{u}}{\partial \nu} \quad \text { on } \quad \Sigma \subset \Gamma .
$$

Then $\Gamma_{0}=\widetilde{\Gamma}_{0}$.

Proof. The proof of the theorem is analogous to the case of bounded regions, see [10], and is based on Holmgren's uniqueness theorem, 
the maximum-minimum principle for the Laplace equation and the analyticity of the solution.

The high instability of the solution with respect to small data perturbations causes ill-posedness of the inverse problem. In order to obtain reliable approximations of the interior boundary curve regularization techniques must be applied. Some methods for the reconstruction of an inclusion in a half plane for the nonstationary heat equation were considered in $[\mathbf{3}, \mathbf{6}]$. They are based on regularized Newton iterations in $[\mathbf{3}]$ and on Landweber iterations in [6]. The numerical experiments have exhibited a slow convergence of the Landweber method in the case of semi-infinite regions. In addition, the solution of direct boundary value problems is required in each iteration step. Furthermore, this method yields inaccurate reconstructions of those parts of the interior curve that have the boundary of the semi-infinite region on their opposite side. As disadvantages of the Newton method, we note that the numerical implementation again requires the solution of a number of Dirichlet problems (1.1)-(1.3) in each iteration and a reasonable a priori information for the initial approximation.

In this paper we consider a method that can be viewed as a hybrid of a decomposition method [7] and a Newton method. This method was first described in [9] and successfully applied for the solution of various inverse boundary value problems in bounded domains [11] and for inverse scattering problems $[\mathbf{5}, \mathbf{1 2}, \mathbf{1 5}]$. It combines the advantages of both Newton and decomposition methods. One of its important properties is that it does not require the solution of direct boundary value problems in each iteration step in contrast to Newton and Landweber methods. This reduces the computational cost and does not lead to a deterioration of the reconstructions as opposed to the decomposition methods, see Section 5.

Our goal in this paper is the extension, mostly numerically, of this hybrid method to semi-infinite regions in $\mathbf{R}^{2}$. The convergence of the hybrid method has only been observed through our numerical experiments. Theoretical investigations on the convergence and applications to other boundary conditions will be the topic of our future research. Note, that the main difficulty in applying the hybrid method for the three-dimensional case is the need of an efficient solver for the corresponding two-dimensional integral equations. 
We consider the following cases of semi-infinite regions:

(i) the strip

$$
D_{1}^{s}:=\left\{x \in \mathbf{R}^{2}: x_{1} \in \mathbf{R}, 0<x_{2}<a, a>0\right\},
$$

(ii) the half strip

$$
D_{1}^{h s}:=\left\{x \in \mathbf{R}^{2}: x_{1}>0,0<x_{2}<a, a>0\right\},
$$

(iii) the upper half plane

$$
D_{1}^{h p}:=\left\{x \in \mathbf{R}^{2}: x_{2}>0\right\}
$$

and the quadrant

$$
D_{1}^{q}:=\left\{x \in \mathbf{R}^{2}: x_{1}>0, x_{2}>0\right\} .
$$

The plan of the paper is as follows. In Section 2 we describe the numerical solution of the direct boundary value problem (1.1)-(1.3) via boundary integral equations of the first kind. For the integral representation of the solution, we use single-layer potentials with the Green function for the respective semi-infinite region. The computation of normal derivatives on the boundary curves is considered in Section 3. The main aspects of applying the hybrid method to our inverse problem are described in Section 4. Finally, in Section 5, results of numerical experiments are presented.

2. A Green's function technique for the solution of the direct problem. The special features of the domain $D$ determine our numerical method for the solution of the direct problem (1.1)-(1.3). Since $D$ is an unbounded domain, the most efficient numerical method is the application of the indirect variant of the boundary integral equation approach. Using the single-layer potential approach with the Green's function $G$ for the domain $D_{1}$, we can seek the solution of the direct problem in the form

$$
\begin{gathered}
(2.1) u(x)=\frac{1}{\omega_{\pi}} \int_{\Gamma_{0}} \varphi(y) G(x, y) d s(y)-\frac{1}{\omega_{\pi}} \int_{\Gamma} f(y) \frac{\partial G(x, y)}{\partial \nu(y)} d s(y), \\
x \in D,
\end{gathered}
$$


with an unknown density $\varphi$ on $\Gamma_{0}$. Here the coefficients are $\omega_{\pi}=2 \pi$ in the case of the domain (1.5) and $\omega_{\pi}=4 \pi$ in the case of the domains (1.6)-(1.8).

Green's function for the Laplace equation in the semi-infinite region $D_{1}$ can be introduced as

$$
G(x, y)=-\ln |x-y|^{2}+g(x, y),
$$

where $g$ is a harmonic and regular function in $D_{1}$ such that $G$ satisfies the homogeneous boundary condition (1.2) with respect to $x$. For the semi-infinite regions (1.5)-(1.8), the function $g$ has the form (see, e.g., $[13])$

$$
\begin{aligned}
g_{s}(x, y) & :=\ln \frac{\left\{\operatorname{ch}\left[\pi\left(x_{1}-y_{1}\right) / a\right]-\cos \left[\pi\left(x_{2}+y_{2}\right) / a\right]\right\}|x-y|^{2}}{\operatorname{ch}\left[\pi\left(x_{1}-y_{1}\right) / a\right]-\cos \left[\pi\left(x_{2}-y_{2}\right) / a\right]}, \quad x \neq y, \\
g_{h s}(x, y) & :=g_{s}(x, y)+\ln \frac{\operatorname{ch}\left[\pi\left(x_{1}+y_{1}\right) / a\right]-\cos \left[\pi\left(x_{2}-y_{2}\right) / a\right]}{\operatorname{ch}\left[\pi\left(x_{1}+y_{1}\right) / a\right]-\cos \left[\pi\left(x_{2}+y_{2}\right) / a\right]}, \quad x \neq y, \\
g_{h p}(x, y) & :=\ln \left[\left(x_{1}-y_{1}\right)^{2}+\left(x_{2}+y_{2}\right)^{2}\right] \\
g_{q}(x, y) & :=\ln \frac{\left[\left(x_{1}+y_{1}\right)^{2}+\left(x_{2}-y_{2}\right)^{2}\right]\left[\left(x_{1}-y_{1}\right)^{2}+\left(x_{2}+y_{2}\right)^{2}\right]}{\left(x_{1}+y_{1}\right)^{2}+\left(x_{2}+y_{2}\right)^{2}},
\end{aligned}
$$

respectively. Here $\operatorname{ch} x:=(\exp (x)+\exp (-x)) / 2$ is the hyperbolic cosine function. Note that the behavior of Green's function at infinity for semi-infinite regions implies the boundedness of the solution in the form (2.1).

By the classical results on the continuity of single-layer potentials and the properties of Green's function, the problem (1.1)-(1.3) is reduced to the integral equation

$$
\frac{1}{\omega_{\pi}} \int_{\Gamma_{0}} \varphi(y) G(x, y) d s(y)=\frac{1}{\omega_{\pi}} \int_{\Gamma} f(y) \frac{\partial G(x, y)}{\partial \nu(y)} d s(y), x \in \Gamma_{0}
$$

of the first kind.

Theorem 2.1. For any given bounded piecewise continuous function $f$, the integral equation (2.2) possesses a unique solution $\varphi \in$ $H^{-1 / 2}\left(\Gamma_{0}\right)$. 
Proof. This theorem is the extension of the classical results from $[\mathbf{8}]$ for the first kind integral equation of potential theory in Sobolev spaces to the semi-infinite case. The restrictions on the boundary function $f$ and the behavior of the normal derivative of Green's function for semiinfinite regions at infinity ensure the existence of the integral on the righthand side in (2.2).

We assume that the boundary curve $\Gamma_{0}$ is given through a parametric representation

$$
\Gamma_{0}:=\left\{x(t)=\left(x_{1}(t), x_{2}(t)\right): 0 \leq t \leq 2 \pi\right\},
$$

where $x: \mathbf{R} \rightarrow \mathbf{R}^{2}$ is $C^{2}$ and $2 \pi$-periodic with $\left|x^{\prime}(t)\right|>0$ for all $t$. Then we can transform (2.2) into the parametric form

$$
\begin{gathered}
\frac{1}{\omega_{\pi}} \int_{0}^{2 \pi} \mu(\tau)\left[-\ln \left(\frac{4}{e} \sin ^{2} \frac{t-\tau}{2}\right)+L(t, \tau)\right] d \tau=w(t) \\
t \in[0,2 \pi]
\end{gathered}
$$

with the $2 \pi$-periodic kernel

$$
L(t, \tau):=G(x(t), x(\tau))+\ln \left(\frac{4}{e} \sin ^{2} \frac{t-\tau}{2}\right) \text { for } t \neq \tau,
$$

the righthand side

$$
w(t):=\frac{1}{\omega_{\pi}} \int_{\Gamma} f(y) \frac{\partial G(x(t), y)}{\partial \nu(y)} d s(y), \quad t \in[0,2 \pi]
$$

and the density $\mu(t):=\varphi(x(t))\left|x^{\prime}(t)\right|$.

Note that the kernel $L$ has the diagonal term

$$
\begin{aligned}
L^{s}(t, t) & =\ln \frac{2 a^{2}\left\{1-\cos \left[(2 \pi / a) x_{2}(t)\right]\right\}}{\pi^{2} e\left|x^{\prime}(t)\right|^{2}}, \\
L^{h s}(t, t) & =L^{s}(t, t)+\ln \frac{\operatorname{ch}\left[(2 \pi / a) x_{1}(t)\right]-1}{\operatorname{ch}\left[(2 \pi / a) x_{1}(t)\right]-\cos \left[(2 \pi / a) x_{2}(t)\right]}, \\
L^{h p}(t, t) & =\ln \frac{1}{e\left|x^{\prime}(t)\right|^{2}}+g_{h p}(x(t), x(t)), \\
L^{q}(t, t) & =\ln \frac{1}{e\left|x^{\prime}(t)\right|^{2}}+g_{q}(x(t), x(t))
\end{aligned}
$$


for the regions (1.5)-(1.8), respectively. The function on the righthand side can be written as

$$
w(t):=\frac{1}{\omega_{\pi}}\left\{\begin{array}{cc}
\sum_{l=1}^{2} \int_{-\infty}^{\infty} f_{l}^{s}(\tau) H_{l}^{s}(t, \tau) d \tau & \text { for } D_{1}^{s}, \\
\sum_{l=1}^{2} \int_{0}^{\infty} f_{l}^{h s}(\tau) H_{l}^{h s}(t, \tau) d \tau & \\
\quad+\int_{0}^{a} f_{3}^{h s}(\tau) H_{3}^{h s}(t, \tau) d \tau & \text { for } D_{1}^{h s}, \\
\int_{-\infty}^{\infty} f^{h p}(\tau) H^{h p}(t, \tau) d \tau & \text { for } D_{1}^{h p}, \\
\sum_{l=1}^{2} \int_{0}^{\infty} f_{l}^{q}(\tau) H_{l}^{q}(t, \tau) d \tau & \text { for } D_{1}^{q} .
\end{array}\right.
$$

Here the functions $H_{l}^{s}, H_{l}^{q}, l=1,2, H_{l}^{h s}, l=1,2,3$, and $H^{h p}$ are obtained by the parameterization of the normal derivative of Green's function on the corresponding parts of the boundary $\Gamma$ for the regions $(1.5)-(1.8)$. They are smooth functions and $f_{l}^{s}, f_{l}^{q}, l=1,2, f_{l}^{h s}$, $l=1,2,3$, and $f^{h p}$ represent the given boundary functions.

For example, in the case of a half strip the kernels $H_{l}^{h s}, l=1,2,3$, in (2.4) have the representations

$$
\begin{aligned}
H_{1}^{h s}(t, \tau):= & \frac{(2 \pi / a) \sin \left[(\pi / a) x_{2}(t)\right]}{\operatorname{ch}\left[(\pi / a)\left(x_{1}(t)+\tau\right)\right]-\cos \left[(\pi / a) x_{2}(t)\right]} \\
& -\frac{(2 \pi / a) \sin \left[(\pi / a) x_{2}(t)\right]}{\operatorname{ch}\left[(\pi / a)\left(x_{1}(t)-\tau\right)\right]-\cos \left[(\pi / a) x_{2}(t)\right]}, \\
H_{2}^{h s}(t, \tau):= & \frac{(2 \pi / a) \sin \left[(\pi / a) x_{2}(t)\right]}{\operatorname{ch}\left[(\pi / a)\left(x_{1}(t)+\tau\right)\right]+\cos \left[(\pi / a) x_{2}(t)\right]} \\
& -\frac{(2 \pi / a) \sin \left[(\pi / a) x_{2}(t)\right]}{\operatorname{ch}\left[(\pi / a)\left(x_{1}(t)-\tau\right)\right]+\cos \left[(\pi / a) x_{2}(t)\right]}, \\
H_{3}^{h s}(t, \tau):= & \frac{(2 \pi / a) \operatorname{sh}\left[(\pi / a) x_{1}(t)\right]}{\operatorname{ch}\left[(\pi / a) x_{1}(t)\right]-\cos \left[(\pi / a)\left(x_{2}(t)+\tau\right)\right]} \\
& -\frac{(2 \pi / a) \operatorname{sh}\left[(\pi / a) x_{1}(t)\right]}{\operatorname{ch}\left[(\pi / a) x_{1}(t)\right]-\cos \left[(\pi / a)\left(x_{2}(t)-\tau\right)\right]} .
\end{aligned}
$$

Here $\operatorname{sh} x:=(\exp (x)-\exp (-x)) / 2$ is the hyperbolic sine function.

For the full discretization of the integral equation of the first kind (2.3) with a logarithmic singularity we apply a collocation method together with quadrature rules $[4,8]$ based on trigonometric interpolation. For this purpose we choose an equidistant mesh by setting

$$
t_{i}:=i \pi / M, \quad i=0, \ldots, 2 M-1, \quad M \in \mathbf{N}
$$


and use the quadrature rules

$$
\frac{1}{2 \pi} \int_{0}^{2 \pi} f(\tau) d \tau \approx \frac{1}{2 M} \sum_{j=0}^{2 M-1} f\left(t_{j}\right)
$$

and

$$
\frac{1}{2 \pi} \int_{0}^{2 \pi} f(\tau) \ln \left(\frac{4}{e} \sin ^{2} \frac{t-\tau}{2}\right) d \tau \approx \sum_{j=0}^{2 M-1} R_{j}(t) f\left(t_{j}\right)
$$

with weight functions

$$
R_{j}(t):=-\frac{1}{2 M}\left\{1+2 \sum_{m=1}^{M-1} \frac{1}{m} \cos m\left(t-t_{j}\right)+\frac{\cos \left(t-t_{j}\right)}{M}\right\} .
$$

These quadrature formulas are obtained by replacing $f$ by its trigonometric interpolation polynomial and then integrating exactly [8]. In the case of periodic analytic functions $f$ we obtain exponential convergence.

For the numerical calculation of the integrals in (2.4) we first transform the integrals over the intervals $(0, \infty)$ and $(0, a)$ to integrals over R. For this we perform the substitutions

$$
\int_{0}^{\infty} f(\tau) d \tau=\int_{-\infty}^{\infty} f\left(e^{\tau}\right) e^{\tau} d \tau
$$

and

$$
\int_{0}^{a} f(\tau) d \tau=a \int_{-\infty}^{\infty} f\left(\frac{a}{e^{-\tau}+1}\right) \frac{e^{-\tau}}{\left(e^{-\tau}+1\right)^{2}} d \tau .
$$

Then the quadrature rule

$$
\begin{gathered}
\int_{-\infty}^{\infty} f(\tau) d \tau \approx h_{\infty} \sum_{i=-M_{1}}^{M_{1}} f\left(i h_{\infty}\right), M_{1} \in \mathbf{N}, \quad h_{\infty}=\frac{c}{\sqrt{M_{1}}} \\
c>0
\end{gathered}
$$


can be applied. This quadrature formula is obtained by replacing $f$ by a sinc approximation, see [16], and then integrating exactly. In the case of analytic functions $f$ satisfying $f(t)=O\left(e^{-c|t|}\right)$ for $|t| \rightarrow \infty$ and some positive constant $c$, the quadrature (2.10) has exponential convergence. In the case of the substitution (2.9), note that if the function $f$ satisfies the property $|f(z)|<c|z|^{\alpha}|a-z|^{\beta}, z \in(0, a)$ for some positive constants $c, \alpha$ and $\beta$, then we have exponential convergence [16] for the quadrature (2.10). Analogously, in the case of the substitution (2.8) algebraic decay of the function $f$ at infinity also ensures exponential convergence.

Thus, after the application of the quadrature rules (2.6) and (2.7) in the integral equation (2.3) and the quadrature rule (2.10) for the computation of the integrals on the righthand side (2.4), we collocate at the quadrature points (2.5) to obtain the system of linear equations

$$
\begin{gathered}
\frac{2 \pi}{\omega_{\pi}} \sum_{j=0}^{2 M-1} \widetilde{\mu}_{j}\left\{R_{j}\left(t_{i}\right)+\frac{1}{2 M} L\left(t_{i}, t_{j}\right)\right\}=\widetilde{w}\left(t_{i}\right) \\
i=0, \ldots, 2 M-1
\end{gathered}
$$

where $\widetilde{\mu}_{j} \approx \mu\left(t_{j}\right)$ and $\widetilde{w}$ is the approximation of (2.4).

A convergence and error analysis for this numerical scheme is described in [4] in a Hölder space setting and in [8] in a Sobolev space setting. This analysis exhibits the dependence of the convergence rate on the smoothness of the boundary curve $\Gamma_{0}$, i.e., the proposed method belongs to the algorithms without "saturation effect."

Finally, for the solution of the direct boundary value problem (1.1)-(1.3), we have the approximation

$$
\widetilde{u}(x)=\frac{2 \pi}{\omega_{\pi}} \cdot \frac{1}{2 M} \sum_{j=0}^{2 M-1} \widetilde{\mu}_{j} G\left(x, x\left(t_{j}\right)\right)-\widetilde{w}_{D}(x), \quad x \in D,
$$

where $\widetilde{w}_{D}$ is calculated analogously to (2.4).

3. The computation of normal derivatives on the boundaries. For the numerical implementation of the inverse problem, we need approximations for the normal derivative of the solution of problem (1.1)-(1.3) on the boundaries $\Gamma_{0}$ and $\Gamma$. From the jump relations for 
the normal derivative of the single- and double-layer potentials in (2.1), see $[8]$, we have

$$
\begin{aligned}
\frac{\partial u}{\partial \nu}(x)= & \frac{1}{2} \varphi(x)+\frac{1}{\omega_{\pi}} \int_{\Gamma_{0}} \varphi(y) \frac{\partial}{\partial \nu(x)} G(x, y) d s(y) \\
& -\frac{1}{\omega_{\pi}} \int_{\Gamma} f(y) \frac{\partial^{2}}{\partial \nu(x) \partial \nu(y)} G(x, y) d s(y), x \in \Gamma_{0}
\end{aligned}
$$

and

$$
\begin{aligned}
\frac{\partial u}{\partial \nu}(x)= & \frac{1}{\omega_{\pi}} \int_{\Gamma_{0}} \varphi(y) \frac{\partial}{\partial \nu(x)} G(x, y) d s(y) \\
& -\frac{1}{\omega_{\pi}} \int_{\Gamma} f(y) \frac{\partial^{2}}{\partial \nu(x) \partial \nu(y)} G(x, y) d s(y), x \in \Gamma
\end{aligned}
$$

After a straightforward calculation of the first and second normal derivatives of Green's function $G$ on the boundary $\Gamma_{0}$ of the inclusion and on the boundary $\Gamma$ of the corresponding semi-infinite region, parameterization and application of the quadrature rules as in the case of the integral equation (2.2), we obtain approximations for the fluxes (3.1) and (3.2).

Let us consider in detail the case when the domain $D_{1}$ is a half strip (1.5). Then the boundary $\Gamma=\Gamma_{1} \cup \Gamma_{2} \cup \Gamma_{3}$, where $\Gamma_{1}:=$ $\left\{\hat{x}_{1}(t)=(t, 0): 0<t<\infty\right\}, \Gamma_{2}:=\left\{\hat{x}_{2}(t)=(t, a): 0<t<\infty\right\}$, $\Gamma_{3}:=\left\{\hat{x}_{3}(t)=(0, t): 0<t<a\right\}$. Concerning formula (3.1), we have the parametric form for the normal derivative on $\Gamma_{0}$ given by

$$
\begin{aligned}
\frac{\partial u}{\partial \nu}(x(t))= & \frac{\mu(t)}{2\left|x^{\prime}(t)\right|}+\frac{1}{\omega_{\pi}} \int_{0}^{2 \pi} \mu(\tau) T_{0}(t, \tau) d \tau \\
& -\frac{1}{\omega_{\pi}} \int_{0}^{\infty} f_{1}(\tau) T_{1}(t, \tau) d \tau \\
& -\frac{1}{\omega_{\pi}} \int_{0}^{\infty} f_{2}(\tau) T_{2}(t, \tau) d \tau \\
& -\frac{1}{\omega_{\pi}} \int_{0}^{a} f_{3}(\tau) T_{3}(t, \tau) d \tau, \quad t \in[0,2 \pi],
\end{aligned}
$$

where $f_{i}(t):=f\left(\widehat{x}_{i}(t)\right), i=1,2,3$, and $\mu$ is the solution of $(2.3)$. 
Here

$$
T_{0}(t, \tau):=\sum_{i=1}^{4} T_{0}^{(i)}(t, \tau)
$$

where

$$
\begin{aligned}
T_{0}^{(1)}(t, \tau):= & \frac{(\pi / a) x_{1}^{\prime}(t) \sin \left[(\pi / a)\left(x_{2}(t)-x_{2}(\tau)\right)\right]}{\left\{\operatorname{ch}\left[(\pi / a)\left(x_{1}(t)+x_{1}(\tau)\right)\right]-\cos \left[(\pi / a)\left(x_{2}(t)-x_{2}(\tau)\right)\right]\right\}\left|x^{\prime}(t)\right|} \\
& -\frac{(\pi / a) x_{2}^{\prime}(t) \operatorname{sh}\left[(\pi / a)\left(x_{1}(t)+x_{1}(\tau)\right)\right]}{\left\{\operatorname{ch}\left[(\pi / a)\left(x_{1}(t)+x_{1}(\tau)\right)\right]-\cos \left[(\pi / a)\left(x_{2}(t)-x_{2}(\tau)\right)\right]\right\}\left|x^{\prime}(t)\right|}, \\
T_{0}^{(2)}(t, \tau):= & \frac{(\pi / a) x_{1}^{\prime}(t) \sin \left[(\pi / a)\left(x_{2}(t)+x_{2}(\tau)\right)\right]-(\pi / a) x_{2}^{\prime}(t) \operatorname{sh}\left[(\pi / a)\left(x_{1}(t)-x_{1}(\tau)\right)\right]}{\left\{\operatorname{ch}\left[(\pi / a)\left(x_{1}(t)-x_{1}(\tau)\right)\right]-\cos \left[(\pi / a)\left(x_{2}(t)+x_{2}(\tau)\right)\right]\right\}\left|x^{\prime}(t)\right|}, \\
T_{0}^{(3)}(t, \tau):= & \frac{(\pi / a) x_{2}^{\prime}(t) \operatorname{sh}\left[(\pi / a)\left(x_{1}(t)+x_{1}(\tau)\right)\right]-(\pi / a) x_{1}^{\prime}(t) \sin \left[(\pi / a)\left(x_{2}(t)+x_{2}(\tau)\right)\right]}{\left\{\operatorname{ch}\left[(\pi / a)\left(x_{1}(t)+x_{1}(\tau)\right)\right]-\cos \left[(\pi / a)\left(x_{2}(t)+x_{2}(\tau)\right)\right]\right\}\left|x^{\prime}(t)\right|} \\
T_{0}^{(4)}(t, \tau):= & t \begin{array}{cc}
\frac{(\pi / a) x_{2}^{\prime}(t) \operatorname{sh}\left[(\pi / a)\left(x_{1}(t)-x_{1}(\tau)\right)\right]}{\left\{\operatorname{ch}\left[(\pi / a)\left(x_{1}(t)-x_{1}(\tau)\right)\right]-\cos \left[(\pi / a)\left(x_{2}(t)-x_{2}(\tau)\right)\right]\right\}\left|x^{\prime}(t)\right|} & t=\tau . \\
-\frac{(\pi / a) x_{1}^{\prime}(t) \sin \left[(\pi / a)\left(x_{2}(t)-x_{2}(\tau)\right)\right]}{\left\{\operatorname{ch}\left[(\pi / a)\left(x_{1}(t)-x_{1}(\tau)\right)\right]-\cos \left[(\pi / a)\left(x_{2}(t)-x_{2}(\tau)\right)\right]\right\}\left|x^{\prime}(t)\right|}, & t \neq \tau, \\
\frac{x_{2}^{\prime \prime}(t) x_{1}^{\prime}(t)-x_{1}^{\prime \prime}(t) x_{2}^{\prime}(t)}{\left|x^{\prime}(t)\right|^{3}}, & t=\tau
\end{array}
\end{aligned}
$$

Let us introduce the functions:

$$
\begin{aligned}
a_{-}^{+}(t, \tau) & :=\frac{2 \pi^{2}}{a^{2}\left|x^{\prime}(t)\right|} \sin \frac{\pi x_{2}(t)}{a} \operatorname{sh}\left[\frac{\pi}{a}\left(x_{1}(t) \pm \tau\right)\right], \\
\left(b_{-}^{+}\right)_{-}^{+}(t, \tau) & :=\frac{2 \pi^{2}}{a^{2}\left|x^{\prime}(t)\right|}\left\{\cos \frac{\pi x_{2}(t)}{a} \operatorname{ch}\left[\frac{\pi}{a}\left(x_{1}(t) \pm \tau\right)\right] \pm 1\right\}, \\
c_{-}^{+}(t, \tau) & :=\frac{2 \pi^{2}}{a^{2}\left|x^{\prime}(t)\right|} \sin \left[\frac{\pi}{a}\left(x_{2}(t) \pm \tau\right)\right] \operatorname{sh} \frac{\pi x_{1}(t)}{a}, \\
d_{-}^{+}(t, \tau) & :=\frac{2 \pi^{2}}{a^{2}\left|x^{\prime}(t)\right|}\left\{1-\operatorname{ch} \frac{\pi x_{1}(t)}{a} \cos \left[\frac{\pi}{a}\left(x_{2}(t) \pm \tau\right)\right]\right\} .
\end{aligned}
$$

Then the functions $T_{1}, T_{2}$ and $T_{3}$ can be represented in the following way:

$$
\begin{aligned}
T_{1}(t, \tau):= & \frac{x_{2}^{\prime}(t) a^{+}(t, \tau)+x_{1}^{\prime}(t)\left(b^{+}\right)_{-}(t, \tau)}{\left\{\operatorname{ch}\left[(\pi / a)\left(x_{1}(t)+\tau\right)\right]-\cos \left[(\pi / a) x_{2}(t)\right]\right\}^{2}} \\
& -\frac{x_{2}^{\prime}(t) a_{-}(t, \tau)+x_{1}^{\prime}(t)\left(b_{-}\right)_{-}(t, \tau)}{\left\{\operatorname{ch}\left[(\pi / a)\left(x_{1}(t)-\tau\right)\right]-\cos \left[(\pi / a) x_{2}(t)\right]\right\}^{2}}, \\
T_{2}(t, \tau):= & \frac{x_{2}^{\prime}(t) a^{+}(t, \tau)+x_{1}^{\prime}(t)\left(b^{+}\right)^{+}(t, \tau)}{\left\{\operatorname{ch}\left[(\pi / a)\left(x_{1}(t)+\tau\right)\right]+\cos \left[(\pi / a) x_{2}(t)\right]\right\}^{2}} \\
& -\frac{x_{2}^{\prime}(t) a_{-}(t, \tau)+x_{1}^{\prime}(t)\left(b_{-}\right)^{+}(t, \tau)}{\left\{\operatorname{ch}\left[(\pi / a)\left(x_{1}(t)-\tau\right)\right]+\cos \left[(\pi / a) x_{2}(t)\right]\right\}^{2}}
\end{aligned}
$$


and

$$
\begin{aligned}
T_{3}(t, \tau):= & \frac{x_{1}^{\prime}(t) c^{+}(t, \tau)+x_{2}^{\prime}(t) d^{+}(t, \tau)}{\left\{\operatorname{ch}\left[(\pi / a)\left(x_{1}(t)+\tau\right)\right]+\cos \left[(\pi / a) x_{2}(t)\right]\right\}^{2}} \\
& -\frac{x_{1}^{\prime}(t) c_{-}(t, \tau)+x_{2}^{\prime}(t) d_{-}(t, \tau)}{\left\{\operatorname{ch}\left[(\pi / a)\left(x_{1}(t)-\tau\right)\right]+\cos \left[(\pi / a) x_{2}(t)\right]\right\}^{2}} .
\end{aligned}
$$

For the discretization of (3.3) we use the quadrature rule (2.6) for the calculation of the integral over the interval $(0,2 \pi)$. For the calculation of the integrals over $(0, \infty)$ and $(0, a)$, we transform them to integrals over $\mathbf{R}$ using the substitutions (2.8) and (2.9), respectively, and then we apply the sinc quadrature rule (2.10).

The flux (3.2) on the boundary $\Gamma$ for the half strip case can be expressed in the form

$$
\begin{aligned}
\frac{\partial u}{\partial \nu}(x)= & \frac{1}{\omega_{\pi}} \int_{\Gamma_{0}} \varphi(y) \frac{\partial G(x, y)}{\partial \nu(x)} d s(y) \\
& -\frac{1}{\omega_{\pi}} \sum_{l=1}^{3} \frac{\partial}{\partial \nu(x)} \int_{\Gamma_{l}} f_{l}(y) \frac{\partial G(x, y)}{\partial \nu(y)} d s(y),
\end{aligned}
$$

where $x \in \Gamma_{k}$ and $f_{k}:=\left.f\right|_{\Gamma_{k}}, k=1,2,3$. After a straightforward calculation of the first and second derivatives of Green's function and parameterization, we have

$$
\begin{aligned}
& \frac{\partial u}{\partial \nu}\left(\widehat{x}_{k}(t)\right)=\frac{1}{\omega_{\pi}} \int_{0}^{2 \pi} \mu(\tau) D_{k}(t, \tau) d \tau \\
& \quad-\frac{1}{\omega_{\pi}} \sum_{l=1}^{2} \int_{0}^{\infty} f_{l}(\tau) D_{k}^{l}(t, \tau) d \tau-\frac{1}{\omega_{\pi}} \int_{0}^{a} f_{3}(\tau) D_{k}^{3}(t, \tau) d \tau
\end{aligned}
$$

for $k=1,2,3$. The kernels can be represented in the following way:

$$
D_{k}(t, \tau):= \begin{cases}\frac{(2 \pi / a) \sin \left[(\pi / a) x_{2}(\tau)\right]}{\operatorname{ch}\left[(\pi / a)\left(t+x_{1}(\tau)\right)\right]-\cos \left[(\pi / a) x_{2}(\tau)\right]} & \\ -\frac{(2 \pi / a) \sin \left[(\pi / a) x_{2}(\tau)\right]}{\operatorname{ch}\left[(\pi / a)\left(t-x_{1}(\tau)\right)\right]-\cos \left[(\pi / a) x_{2}(\tau)\right]}, & k=1, \\ \frac{(2 \pi / a) \sin \left[(\pi / a) x_{2}(\tau)\right]}{\operatorname{ch}\left[(\pi / a)\left(t+x_{1}(\tau)\right)\right]+\cos \left[(\pi / a) x_{2}(\tau)\right]} & \\ -\frac{(2 \pi / a) \sin \left[(\pi / a) x_{2}(\tau)\right]}{\operatorname{ch}\left[(\pi / a)\left(t-x_{1}(\tau)\right)\right]+\cos \left[(\pi / a) x_{2}(\tau)\right]}, & k=2, \\ \frac{(2 \pi / a) \operatorname{sh}\left[(\pi / a) x_{1}(\tau)\right]}{\operatorname{ch}\left[(\pi / a) x_{1}(\tau)\right]-\cos \left[(\pi / a)\left(t+x_{2}(\tau)\right)\right]} & \\ -\frac{(2 \pi / a) \operatorname{sh}\left[(\pi / a) x_{1}(\tau)\right]}{\operatorname{ch}\left[(\pi / a) x_{1}(\tau)\right]-\cos \left[(\pi / a)\left(t-x_{2}(\tau)\right)\right]}, & k=3,\end{cases}
$$




$$
\begin{aligned}
D_{1}^{1}(t, \tau) & =D_{2}^{2}(t, \tau) \\
& :=\frac{2 \pi^{2}}{a^{2}}\left\{\frac{1}{\operatorname{ch}[(\pi / a)(t-\tau)]-1}-\frac{1}{\operatorname{ch}[(\pi / a)(t+\tau)]-1}\right\} \\
D_{1}^{2}(t, \tau) & =D_{2}^{1}(t, \tau) \\
& :=\frac{2 \pi^{2}}{a^{2}}\left\{\frac{1}{\operatorname{ch}[(\pi / a)(t-\tau)]+1}-\frac{1}{\operatorname{ch}[(\pi / a)(t+\tau)]+1}\right\} \\
D_{1}^{3}(t, \tau) & :=\frac{4 \pi^{2} \sin (\pi \tau / a) \operatorname{sh}(\pi t / a)}{a^{2}(\operatorname{ch}(\pi t / a)-\cos (\pi \tau / a))^{2}}, \quad D_{3}^{1}(t, \tau):=D_{1}^{3}(\tau, t), \\
D_{2}^{3}(t, \tau) & :=\frac{4 \pi^{2} \sin (\pi \tau / a) \operatorname{sh}(\pi t / a)}{a^{2}(\operatorname{ch}(\pi t / a)+\cos (\pi \tau / a))^{2}}, \quad D_{3}^{2}(t, \tau):=D_{2}^{3}(\tau, t), \\
D_{3}^{3}(t, \tau) & :=\frac{2 \pi^{2}}{a^{2}}\left\{\frac{1}{1-\cos [(\pi / a)(t-\tau)]}-\frac{1}{1-\cos [(\pi / a)(t+\tau)]}\right\} .
\end{aligned}
$$

Clearly, we can write the kernels $D_{i}^{i}$ in the form

$$
D_{i}^{i}(t, \tau)=\frac{2 \pi^{2}}{a^{2}}\left\{D_{i}^{i, 1}(t, \tau)-D_{i}^{i, 2}(t, \tau)\right\}, \quad i=1,2,3 .
$$

The numerical calculation of the flux (3.4) causes additional difficulties because of a hypersingularity in the kernels $D_{i}^{i, 1}, i=1,2,3$. For this purpose, we apply a subtraction of a singularity via

$$
\int_{0}^{\infty} f_{i}(\tau) D_{i}^{i, 1}(t, \tau) d \tau=\int_{-\infty}^{\infty} \hat{f}_{i}(\tau) \widehat{D}_{i}^{i}(t, \tau) \frac{1}{(t-\tau)^{2}} d \tau, \quad i=1,2
$$

and

$$
\int_{0}^{a} f_{i}(\tau) D_{3}^{3,1}(t, \tau) d \tau=\int_{-\infty}^{\infty} \hat{f}_{3}(\tau) \widehat{D}_{3}^{3}(t, \tau) \frac{1}{(t-\tau)^{2}} d \tau .
$$

Here $\hat{f}_{i}(t):=f_{i}\left(\psi_{i}(t)\right), \widehat{D}_{i}^{i}(t, \tau):=D_{i}^{i, 1}\left(\psi_{i}(t), \psi_{i}(\tau)\right) \psi_{i}^{\prime}(\tau)(t-\tau)^{2}$ for $t \neq \tau, i=1,2,3$, with the diagonal terms

$$
\begin{aligned}
& \widehat{D}_{i}^{i}(t, t)=\frac{2 a^{2} e^{-t}}{\pi^{2}}, \quad i=1,2, \\
& \widehat{D}_{3}^{3}(t, t)=\frac{2 a\left(e^{-t}+1\right)^{2}}{\pi^{2} e^{-t}}, \quad t \in \mathbf{R} .
\end{aligned}
$$


The functions $\psi_{i}$ are given as

$$
\psi_{i}(t):= \begin{cases}e^{t} & \text { for } i=1,2 \\ a e^{t} /\left(e^{t}+1\right) & \text { for } i=3\end{cases}
$$

By partial integration using the behavior of the kernels at infinity we reduce the Hadamard finite-part integrals to the Cauchy principal value integrals

$$
\begin{gathered}
\int_{-\infty}^{\infty} \hat{f}_{i}(\tau) \widehat{D}_{i}^{i}(t, \tau) \frac{1}{(t-\tau)^{2}} d \tau=\int_{-\infty}^{\infty}\left(\hat{f}_{i}(\tau) \widehat{D}_{i}^{i}(t, \tau)\right)_{\tau}^{\prime} \frac{1}{t-\tau} d \tau \\
t \in \mathbf{R}, \quad i=1,2,3
\end{gathered}
$$

For their numerical integration we use the sinc quadrature rule

$$
\frac{1}{\pi} \int_{-\infty}^{\infty} \frac{f^{\prime}(\tau)}{t-\tau} d \tau \approx \sum_{i=-M_{1}}^{M_{1}} f\left(i h_{\infty}\right) R_{i, \infty}(t), \quad t \in \mathbf{R}
$$

where the weight functions have the form

$$
R_{i, \infty}(t):=\sum_{k=-M_{1}, k \neq i}^{M_{1}} \frac{(-1)^{(k-i)}}{k-i} \cdot \frac{1-\cos \left[\left(\pi / h_{\infty}\right)\left(t-i h_{\infty}\right)\right]}{\left(\pi / h_{\infty}\right)\left(t-i h_{\infty}\right)} .
$$

This quadrature is obtained via an approximation of the derivative $f^{\prime}$ in (3.5) by the cardinal function [16] and exact integration. The convergence rate of this formula is $O\left(M_{1}^{3 / 2} e^{-c M_{1}^{1 / 2}}\right), c>0$.

Now applying the corresponding quadrature formulas in (3.4) we receive an approximate flux on $\Gamma_{k}, k=1,2,3$. For the numerical calculation of the integrals over the interval $(0,2 \pi)$, we use the quadrature rule (2.6). For the calculation of the integrals without singularities on $(0, \infty)$ and $(0, a)$, we transform them to integrals over $\mathbf{R}$ using the substitutions (2.8) and (2.9), respectively, and then we apply the sinc quadrature rule (2.10). The integrals with the hypersingularity are calculated by formula (3.5) after making the corresponding transformations.

4. Hybrid method for the inverse problem. The inverse problem (1.1)-(1.4) defines the nonlinear operator $A$ that maps the 
boundary $\Gamma_{0}$ to the Dirichlet data $u$ on $\Gamma_{0}$ for fixed $\Gamma$ and given Cauchy data $f$ and $\phi$. Thus, the inverse problem can be written in the form of a nonlinear equation

$$
A\left(\Gamma_{0}\right)=0 .
$$

Assume that the boundary curve $\Gamma_{0}$ has the parameterization $\Gamma_{0}=$ $\{x(t), t \in[0,2 \pi]\}$ with a $2 \pi$-periodic function $x: \mathbf{R} \rightarrow \mathbf{R}^{2}$.

For the linearization of the mapping $A$, we first show differentiability and develop a representation of the Fréchet derivative of $A$.

Theorem 4.1. The operator $A: C^{2}[0,2 \pi] \rightarrow C[0,2 \pi]$ is Fréchet differentiable, and its derivative is given by

$$
A^{\prime}(x) h=\langle\operatorname{grad} u \circ x, h\rangle,
$$

where $h \in C^{2}$ is some small perturbation in $\mathbf{R}^{2}$ and $\langle\cdot, \cdot\rangle$ defines the scalar product in $\mathbf{R}^{2}$.

Proof. Analogously to $[\mathbf{1 2}, \mathbf{1 5}]$, we use the definition of the Fréchet derivative. Let $h$ be sufficiently small to ensure that the parameterization $\Gamma_{0, h}=\{x(t)+h(t), t \in[0,2 \pi]\}$ describes a smooth closed curve $\Gamma_{0, h} \in D_{1}$. By using Taylor's formula,

$$
u(x+h)(t)=u(x(t))+\langle\operatorname{grad} u(x(t)), h(t)\rangle+O\left(|h|^{2}\right), \quad t \in[0,2 \pi],
$$

we have

$A(x+h)-A(x)=u \circ(x+h)-u \circ x=\langle\operatorname{grad} u(x(t)), h(t)\rangle+O\left(|h|^{2}\right)$.

Thus, we obtain

$$
\left\|A(x+h)-A(x)-A^{\prime}(x) h\right\|=O\left(\|h\|_{C^{2}}\right), \quad\|h\|_{C^{2}} \rightarrow 0 .
$$

The statement of the theorem follows from the definition of the Fréchet derivative.

We consider the case of starlike boundaries, i.e,

$$
\Gamma_{0}=\left\{x(t)=\left(r(t) \cos t+d_{1}, r(t) \sin t+d_{2}\right): 0 \leq t \leq 2 \pi\right\}
$$


with center $\left(d_{1}, d_{2}\right)$ and an unknown radial function $r \in C^{2}[0,2 \pi]$. Then the nonlinear equation (4.1) has the following parametric form

$$
\hat{A}(r)=0 \text {. }
$$

The Newton method applied to (4.3) yields the linear equation

$$
\hat{A}^{\prime}(r) q+\hat{A}(r)=0
$$

with a fixed function $r$, a correction term $q$ and the Fréchet derivative $\hat{A}^{\prime}$ of the operator $\hat{A}$. Using the results of Theorem 4.1, the operator $\hat{A}^{\prime}$ can be represented as

$$
\hat{A}^{\prime}(r) q=\frac{\partial u}{\partial \theta} q
$$

with the derivative $\partial u / \partial \theta$ in the radial direction. It is necessary to solve the linear equation

$$
\frac{\partial u}{\partial \theta} q=-u \quad \text { on } \quad \Gamma_{0}
$$

for the correction $q$ in each Newton iteration.

Let us now describe the algorithm of the hybrid method in detail.

1. For a given radial function $r$, we solve the integral equation of the first kind

$$
\begin{aligned}
& \frac{1}{\omega_{\pi}} \int_{\Gamma_{0}} \varphi(y) \frac{\partial G(x, y)}{\partial \nu(x)} d s(y) \\
&=\phi(x)+\frac{1}{\omega_{\pi}} \frac{\partial}{\partial \nu(x)} \int_{\Gamma} f(y) \frac{\partial G(x, y)}{\partial \nu(y)} d s(y), \quad x \in \Sigma
\end{aligned}
$$

with unknown density $\varphi$ by the quadrature method. In [5] it was shown that the normal derivative of the single-layer potential defines an operator from $L^{2}\left(\Gamma_{0}\right)$ to $L^{2}(\Gamma)$ that is injective and has dense range. Thus, the application of Tikhonov regularization to the integral equation (4.6) is possible.

Connecting formula (4.6) with the investigations that were made in Section 3 , we get the system of linear equations

$$
\frac{2 \pi}{\omega_{\pi}} \cdot \frac{1}{2 M} \sum_{j=0}^{2 M-1} \widetilde{\mu}_{j} \frac{\partial G\left(x\left(\hat{t}_{i}\right), x\left(t_{j}\right)\right)}{\partial \nu\left(x\left(\hat{t}_{i}\right)\right)}=F\left(\hat{t}_{i}\right),
$$


where $F$ is a known righthand side, $\widehat{t_{i}}, i=0, \ldots, \widehat{M}, \widehat{M} \in \mathbf{N}$ are the points of an equidistant mesh on $\Sigma$ with step size $h=|\Sigma| / \hat{M}$, $\widehat{M}>0$, and $t_{j}, j=0, \ldots, 2 M-1$ are the points for the trigonometric interpolation (2.5).

Since $\widehat{M} \gg 2 M-1$ and due to the ill-posedness the overdetermined system (4.7) is solved by the least squares method with Tikhonov regularization with some regularization parameter $\alpha>0$.

2. The potential approach (2.1) is used to find the function $u$ on $\Gamma_{0}$, taking into account that the kernel $G(x(t), x(\tau))$ has a logarithmic singularity when $t \rightarrow \tau$. For the numerical approximation of $u$ on $\Gamma_{0}$ we obtain

$$
\begin{gathered}
\widetilde{u}\left(x\left(t_{i}\right)\right)=\frac{2 \pi}{\omega_{\pi}} \sum_{j=0}^{2 M-1} \widetilde{\mu}_{j}\left\{-R_{j}\left(t_{i}\right)+\frac{1}{2 M} L\left(t_{i}, t_{j}\right)\right\}-\tilde{w}\left(t_{i}\right), \\
x\left(t_{i}\right) \in \Gamma_{0}, \quad i=0, \ldots, 2 M-1 .
\end{gathered}
$$

3. The derivative $\partial u / \partial \theta$ is computed with the aid of the relation

$$
\frac{\partial u(x)}{\partial \theta}=\frac{\partial u(x)}{\partial \nu}\langle\theta, \nu\rangle+\frac{\partial u(x)}{\partial \vartheta}\langle\theta, \vartheta\rangle, \quad x \in \Gamma_{0},
$$

where $\vartheta$ is the unit tangential vector. Here the approximation of $\partial u(x) / \partial \nu$ on $\Gamma_{0}$ is calculated by formula (3.1) and the tangential derivative $\partial u(x) / \partial \vartheta$ by trigonometric interpolation

$$
\frac{\partial \widetilde{u}}{\partial \vartheta}(x(t))=\frac{1}{\left|x^{\prime}(t)\right|} \sum_{i=0}^{2 M-1} \widetilde{u}\left(t_{i}\right) l_{i}^{\prime}(t),
$$

where $l_{i}, i=0, \ldots, 2 M-1$ are the trigonometric Lagrange basis functions [8].

We can evaluate the approximation of $\partial \widetilde{u} / \partial \theta\left(s_{k}\right)$ at the points $s_{k}=$ $\left(\cos t_{k}, \sin t_{k}\right), k=0, \ldots, 2 M-1$, by formula (4.8).

4. For the numerical solution of the linear equation (4.5), a collocation method is used. We approximate the function $q$ by

$$
\tilde{q}=\sum_{k=1}^{K} a_{k} q_{k}
$$




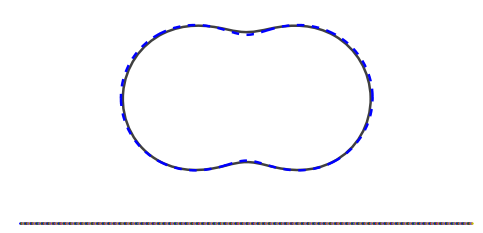

no data noise

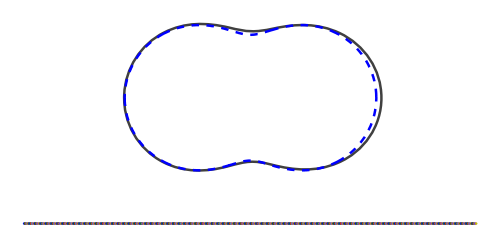

$3 \%$ data noise

FIGURE 1. Reconstruction of the peanut in the upper half plane.

with unknown coefficients $a_{k}$ and given basis functions $q_{k}$ and have to solve the linear system

$$
\sum_{k=1}^{K} a_{k} \frac{\partial \widetilde{u}\left(x\left(t_{i}\right)\right)}{\partial \theta\left(s_{i}\right)} q_{k}\left(t_{i}\right)=-\widetilde{u}\left(x\left(t_{i}\right)\right), \quad i=0, \ldots, 2 M-1 .
$$

Since $2 M-1 \gg K$ and due to the ill-posedness the over-determined system, (4.9) is solved by the least squares method with Tikhonov regularization. Thus, we minimize the penalized residual

$$
\begin{aligned}
\mathcal{F}\left(a_{1}, \ldots, a_{K}\right)= & \lambda \sum_{k=1}^{K}\left(1+k^{2}\right)^{l} a_{k}^{2} \\
& +\sum_{i=0}^{2 M-1}\left|\sum_{k=1}^{K} a_{k} \frac{\partial \widetilde{u}\left(x\left(t_{i}\right)\right)}{\partial \theta} q_{k}\left(t_{i}\right)+\widetilde{u}\left(x\left(t_{i}\right)\right)\right|^{2}
\end{aligned}
$$

with some regularization parameter $\lambda>0$ and some moderately large Sobolev index $l$. The minimization of $\mathcal{F}$ with respect to $a_{1}, \ldots, a_{K}$ is equivalent to solving the normal equations.

5. Numerical examples. In our numerical experiments we investigate the feasibility of the hybrid method by means of two cases. First, using synthetic data, i.e., creating the flux $\phi=\partial u / \partial \nu$ by solving the direct boundary value problem for a known interior radial function, we test the quality of the reconstruction. In the second case we find the approximation of the boundary for noisy data. Here random pointwise 


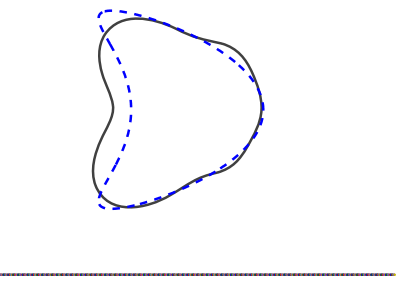

no data noise

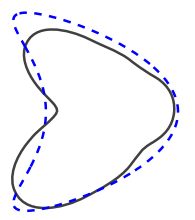

$3 \%$ data noise

FIGURE 2. Reconstruction of the kite in the upper half plane.

errors have been added to the values of $\phi$, with the percentage given in terms of the $L^{2}$ norm. We reconstruct inclusions that belong to the class of starlike boundaries. As an approximating subspace for the radial functions we choose trigonometric polynomials of degree less than or equal to $K$, i.e.,

$$
\tilde{q}(t)=\sum_{k=0}^{K} a_{k} \cos k t+\sum_{k=K+1}^{2 K} a_{k} \sin (k-K) t .
$$

1. The upper half plane case. First we consider the identification of a peanut-shaped boundary curve $\Gamma_{0}$ with the center coordinates $d_{1}=0$ and $d_{2}=1$ in (4.2) and the radial function

$$
r(t)=\sqrt{\cos ^{2} t+0.25 \sin ^{2} t}, \quad 0 \leq t \leq 2 \pi
$$

in the upper half plane $x_{2}>0$. The boundary function on $\Gamma=$ $\{(t, 0), t \in \mathbf{R}\}$ is

$$
f(t)=\exp \left(-t^{2}\right), \quad t \in \mathbf{R} .
$$

For this test we use the following parameters: the number of collocation points $M=16$, the number of collocation points for flux measurement $\widehat{M}=6 M$, the number of sinc quadrature points $M_{1}=2000$, the degree for the basis trigonometric functions $K=4$, the Sobolev index $l=2$. The flux on the boundary of the half plane is calculated on $\Sigma=\{(t, 0),-30<t<30\}$. As an initial guess we choose the circle 


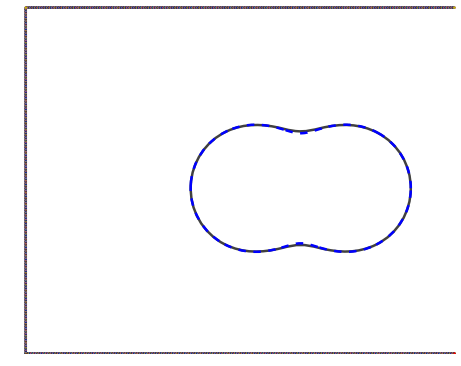

no data noise

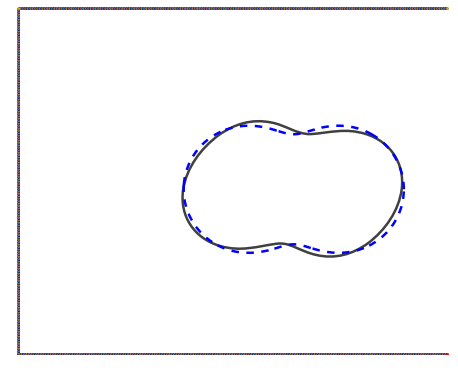

$3 \%$ data noise

FIGURE 3. Reconstruction of the peanut in the half strip.

with radius $R=1$. The results after nine iteration steps are presented in Figure 1. In the case of exact input data we use the regularization parameters $\alpha=10^{-9}, \lambda=0.1$, and in the case of noisy data $\alpha=0.001$ and $\lambda=0.5$.

In the second example we consider the reconstruction of a kite shaped boundary curve with the parameterization

$$
\begin{array}{r}
\Gamma_{0}=\left\{x(t)=\left(0.6 \cos t+0.39 \cos 2 t-0.39+d_{1}, 0.9 \sin t+d_{2}\right),\right. \\
0 \leq t \leq 2 \pi\}
\end{array}
$$

and the boundary function

$$
f(t)=1, \quad t \in \mathbf{R}
$$

The results of the numerical experiments after 19 iteration steps are shown in Figure 2. Here we use the following parameters: $d_{1}=0$, $d_{2}=1.5, M=16, \widehat{M}=6 M, M_{1}=2000, K=6, l=2, \alpha=10^{-4}$, $\lambda=0.1$. The observation boundary $\Sigma$ is the same as in the previous case. 


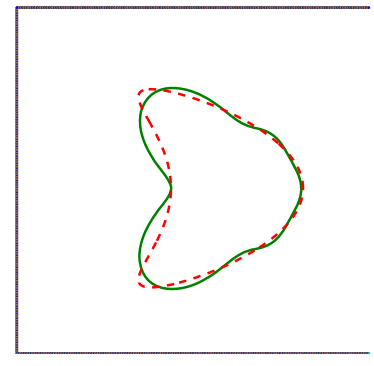

no data noise

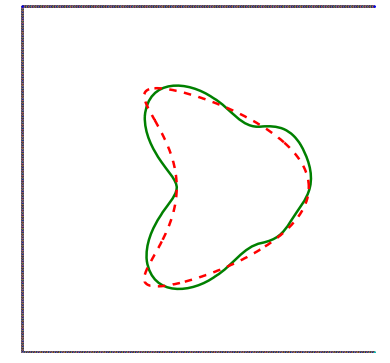

$3 \%$ data noise

FIGURE 4. Reconstruction of the kite in the half strip.

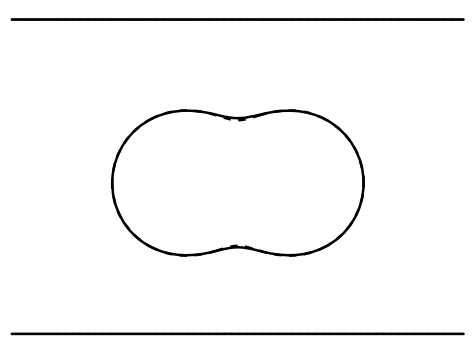

no data noise

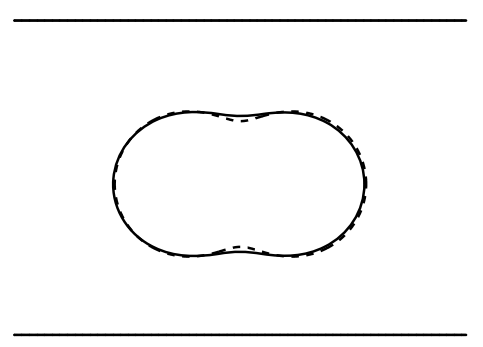

$3 \%$ data noise

FIGURE 5. Reconstruction of the peanut in the strip.

2. Half strip case. Here we also consider two variants of inclusions. First we reconstruct the peanut shaped inclusion with the radial function (5.1) in the half strip region with $d_{1}=2.5$ and $d_{2}=1.5$ and the boundary function (5.3). The width of the half strip is $a=\pi$. The necessary parameters are chosen as follows: $M=16, M_{1}=500$, $K=6$ and $l=2$. The flux is given on $\Sigma=\Sigma_{1} \cup \Sigma_{2} \cup \Sigma_{2}$, where $\Sigma_{1}:=\{(t, 0), 0.5 \leq t \leq 6\}$ with $\widehat{M}_{1}=M, \Sigma_{2}:=\{(t, a), 0.5 \leq t \leq 6\}$ with $\widehat{M}_{2}=M$ and $\Sigma_{3}:=\{(0, t), 0.5 \leq t \leq 2.5\}$ with $\widehat{M}_{3}=M$, $\widehat{M}=\widehat{M}_{1}+\widehat{M}_{2}+\widehat{M}_{3}$. 
The results after 9 iterations are presented in Figure 3 . The regularization parameters are $\alpha=10^{-6}, \lambda=10^{-4}$ in the case of exact data and $\alpha=10^{-4}, \lambda=10^{-2}$ in the case of 3 percent data noise.

For the kite reconstruction in the half strip with the boundary data

$$
f(t)=\exp \left(-\sin ^{2} t\right), \quad t \in \mathbf{R}
$$

we use $\alpha=0.001, \lambda=0.01$. The results are presented in Figure 4 . The other parameters are the same as in the previous test.

3. Strip case. For the reconstruction of the peanut in the strip with $d_{1}=0$ and $d_{2}=1.5$, we use $\alpha=10^{-9}, \lambda=0.1$ in the case of exact data and $\alpha=0.01, \lambda=0.5$ in the case of 3 percent data noise. The width of the strip is $a=2.5$ and the flux is given on $\Sigma:=\Sigma_{1} \cup \Sigma_{2}$. The other parameters are the same as in the case of the half strip. The results are presented in Figure 5.

As one can observe, the numerical tests indicate a satisfactory boundary reconstruction quality for the inclusions with a reasonable stability against noisy data.

\section{REFERENCES}

1. N.D. Aparicio and M.K. Pidock, The boundary inverse problem for the Laplace equation in two dimensions, Inverse Problems 12 (1996), 565-577.

2. H.T. Banks and F. Kojima, Boundary shape identification in two-dimensional electrostatic problems using SQUIDs, J. Ill-Posed Inverse Problems 8 (2000), 467-504.

3. R. Chapko, On the numerical solution of direct and inverse problems for the heat equation in a semi-infinite region, J. Computational Appl. Math. 108 (1999), 41-55.

4. R. Chapko and R. Kress, On a quadrature method for a logarithmic integral equation of the first kind, in World scientific series in applicable analysis, Vol. 2, Agarwal, ed., World Scientific, Singapore, 1993.

5. — A hybrid method for inverse boundary value problems in potential theory, J. Ill-Posed Inverse Problems 13 (2005), 27-40.

6. R. Chapko and N. Vintonyak, On Landweber and integral equation methods for inverse boundary value problem of nonstationary heat conductivity in a semiinfinite domain, Visnyk Lviv. Universytetu. Ser. prykl. matem. ta informatyka. 8 (2004), 77-90 (in Ukrainian).

7. A. Kirsch and R. Kress, An optimization method in inverse acoustic scattering, in Boundary element IX, Vol. 3, Springer-Verlag, Berlin, 1987. 
8. R. Kress, Linear integral equations, 2nd. ed., Springer-Verlag, Heidelberg, 1999.

9. - Newton's method for inverse obstacle scattering meets the method of least squares, Inverse Problems 19 (2003), 91-104.

10. - Inverse Dirichlet problem and conformal mapping, Math. Comp. Simu. 66 (2004), 255-265.

11. R. Kress and P. Serranho, A hybrid method for two-dimensional crack reconstruction, Inverse Problems 21 (2005), 773-784.

12. - A hybrid method for sound-hard obstacle reconstruction, J. Comput. Appl. Math., to appear.

13. A. Polyanin, Handbook of linear partial differential equations for engineers and scientists, Chapman \& Hall, CRC Press, Boca Raton, 2002.

14. J.R. Roche and J. Sokolowski, Numerical methods for shape identification problems, Control Cybernetics 25 (1996), 867-894.

15. P. Serranho, A hybrid method for inverse scattering for shape and impedance, Inverse Problems 22 (2006), 663-680.

16. F. Stenger, Numerical methods based on sinc and analytic functions, Springer-Verlag, Heidelberg, 1993.

Faculty of Applied Mathematics and Computer Science, Ivan Franko National University of LViv, 79000 Lviv, Ukraine

Email address: chapko@is.lviv.ua

Faculty of Applied Mathematics and Computer Science, Ivan Franko National University of Lviv, 79000 Lviv, Ukraine

Email address: nadia_vintonyak@ukr.net 